\title{
Microscope Image Processing: An Overview
}

\author{
N.M. Chaudhari \\ Department of Computer Engineering \\ KCE'S College of Engineering \& IT \\ Jalgaon (M.S.), India
}

\author{
B.V. Pawar \\ School of Computer Science \\ North Maharashtra University \\ Jalgaon (M.S.), India
}

\begin{abstract}
Microscope Image Processing is an area of digital image processing in which most of image processing techniques are applied on the image captured by the microscope. Due to technical advances in microscope, it becomes easy to interface microscope to image processing system. Most of the fields like medicine, biological research, test tube baby, cancer research, drug testing, metallurgy, etc need this type of processing for the analysis purpose. This paper covers the overview of Microscope Image processing and a generic Microscope Image processing system. The concept of microscope image acquisition, various techniques and its analysis is also discussed here. Further it includes various application areas of microscope image processing. There is call for researcher to pay attention on Wikipedia in this area, which is emerging field in image processing.
\end{abstract}

\section{Keywords}

Microscope image processing, digital image processing, image analysis

\section{INTRODUCTION}

Microscope image processing (MIP) has seen increasingly used in biomedical research and clinical medicine as well as in many other fields during last few years. This is due to many variations of the basic microscope instruments which allow us to peer into spaces much too small to be seen with the solo eye.

Digital image processing greatly enriches the process of extracting information about the specimen from a microscope image. Therefore digital imaging is steadily becoming an integral part of microscopy.

Microscope image processing can be used to mine quantitative information about the specimen from a microscope image, and it can transform an image so that a displayed version is much more informative than the original one.

\section{WHAT IS MICROSCOPE IMAGE PROCESSING?}

The images produced by a microscope are easily converted into digital form for storage, its analysis, or processing an image prior to display and interpretation is called Microscope Image Processing (MIP). Digitization process forms the digital image from the optical image which is obtained from microscope. Microscopes have long been used to capture, observe, measure, and analyze the images of various living organisms and structures at scales far below normal human visual perception. With the advent of affordable, high-performance computer and image sensor technologies, digital imaging has come into eminent and is replacing traditional film-based photomicrography as the most widely used method for microscope image acquisition and storage.

Digital image processing is not only a natural extension but is proving to be essential to the success of subsequent data analysis and interpretation of the new generation of microscope images. There is microscope imaging modalities where an image suitable for viewing is only available after digital image processing. Digital processing of microscope images has opened up new lands of medical research. This can be used for better analysis in clinical diagnostic procedures which helps practitioners to take right decisions in critical cases [1].

\section{MICROSCOPE IMAGE PROCESSING SYSTEM}

A typical microscope image processing system consists of three simple steps. First image is acquired through microscope, then various Images processing techniques will applied on it. This data is further used for the analysis purpose. The microscope image processing system is shown in figure 1 .

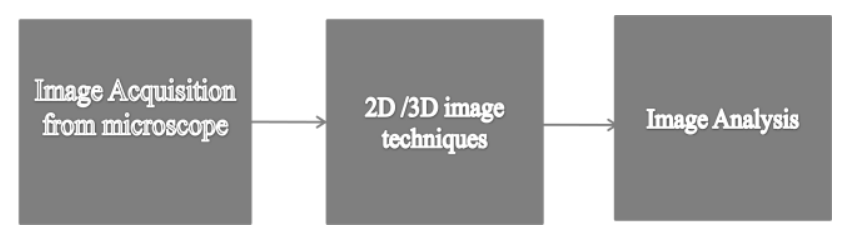

Figure 1: A General Microscopic Image Processing System

\subsection{Image Acquisition From Microscope}

In modern days, acquisition is usually done using a inverted camera mounted in the optical path of the microscope. This type of imaging system allows real-time visualization of the plate being examined in a computer monitor or in a television set. The camera may be full color or monochrome with very high resolution so as to gain as much direct information as possible. Sometimes digital cameras are used for this application

While acquiring the data one can take still photograph or can record video with 25-30 frames per second or higher. Acquisition with high rate and high resolution detector allows dynamic processes to be observed in real time, which can be stored for later analysis. Combined with the high image resolution, this approach can generate vast quantities of raw data, which can be a challenge to deal with. Another challenge is poor Signal to Noise Ratio (SNR).

As per the application requirements one can acquire the image in spite of lower resolution detector which has higher acquisition rate, for faster events. If the object is motionless one can acquire the image with highest possible spatial resolution. [1].

\subsection{D/3D IMAGE TECHNIQUES}

As same as traditional image processing the microscopic application also used fundamental techniques to produce the information contain in the microscopic sample. Due to the distortion caused by the camera and eye hole and the illumination effect one need to adjust the brightness, contrast of image. Also first step image averaging need to apply to reduce 
the noise and image correction. Most of the microscopic 2D operations are discussed in section 5. Image convolution, de convolution and other highly developed techniques are used to sharpen the image. For 3D image analysis one need to take a series of images at a fixed position, but at dissimilar focal depths. Since the majority microscopic samples are essentially transparent, and the depth of field of the focused sample is exceptionally narrow, it is possible to capture images through a three-dimensional object using 2D equipment like confocal microscopes. Software is then able to reconstruct a 3D model of the original sample which may be manipulated correctly [1].

\subsection{Image Analysis}

Lower level image processing techniques are applicable for image analysis. It includes finding the edges of object, object count, classify the object, measurement of object. It is possible to label the object and track the motion of the labeled object in the series of frames [1].

\section{MEASUREMENT OF MICROSCOPE OBJECTS}

Microscope may be equipped with a scale (called a reticule) that is built into one eyepiece. The reticule can be used to measure any planar dimension in a microscope field since the ocular can be turned in any direction and the object of interest can be repositioned with the stage manipulators. To measure the lengths of an object note the number of ocular divisions spanned by the object. Then multiply by the conversion factor for the magnification used. The conversion factor is different at each magnification. Therefore, when using a reticule for the first time, it is necessary to calibrate the scale by focusing on a second micrometer scale (a stage micrometer) placed directly on the stage.

\subsection{Conversion factor}

Identify the ocular micrometer. A usual scale consists of 50 100 divisions. Fine-tuning of the focus of eyepiece in order to make the scale as sharp as possible, also adjust the other eyepiece to match the focus. Any ocular scale must be calibrated, using a device called a stage micrometer. A stage micrometer is simply a microscope slide with a scale etched on the surface as shown in figure 2. A typical micrometer scale is $2 \mathrm{~mm}$ long and at least part of it should be engraved with divisions of $0.01 \mathrm{~mm}(10 \mu \mathrm{m})$. Suppose that a stage micrometer scale has divisions that are equal to $0.1 \mathrm{~mm}$, which is 100 micrometers $(\mu \mathrm{m})$. Suppose that the scale is lined up with the ocular scale, and at $100 \mathrm{X}$ it is observed that each micrometer division covers the same distance as 10 ocular divisions. Then one ocular division (smallest increment on the scale) $=10 \mu \mathrm{m}$ at 100 power. The conversion to other magnifications is accomplished by factoring in the difference in magnification. In the illustration, the calibration would be 25 $\mu \mathrm{m}$ at $40 \mathrm{X}, 2.5 \mu \mathrm{m}$ at $400 \mathrm{X}$, and $1 \mu \mathrm{m}$ at $1000 \mathrm{X}$.

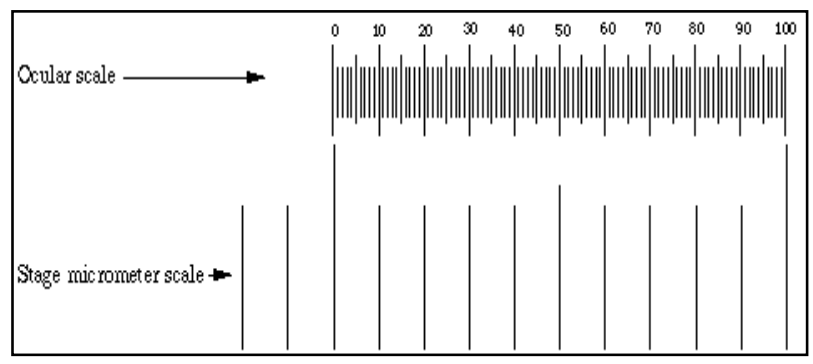

Figure 2: Typical view of Micrometer Scale
As shown in figure 3 some stage micrometers are finely divided only at one end so that the measurement of the diameter of microscope field is done in more precisely. One of the larger divisions is positioned at one edge of the field of view, so that the fine parts of the scale is overlapping the opposite side. Thus the field diameter can be determined to the optimum available precision [2].

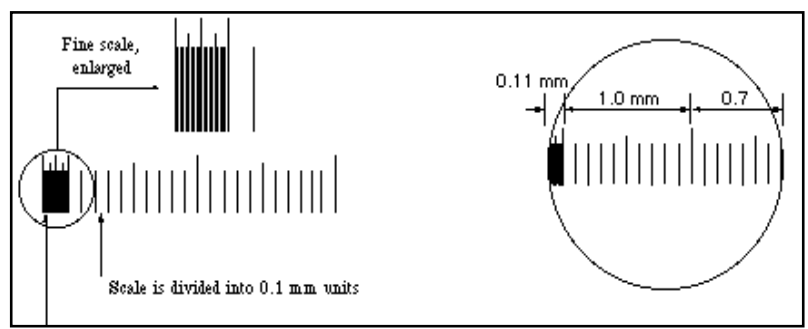

Figure 3: Field view of Stage micrometer

\section{MICROSCOPE IMAGE PROCESSING TECHNIQUES}

While acquiring the image, the distortion and blurring caused by the microscope instrument and adjustment of illumination for brightness and contrast, one need to enhance the image for the analysis. To reduce this noise image averaging and image corrections are often used. Most of the techniques used for enhancement, segmentation, object measurement and classification are discussed here. Thanks to authors Qiang $\mathrm{Wu}$, et.al for introducing this techniques specific for microscopic images [3].

\subsection{Image enhancement}

Image enhancement is the process of enhancing the appearance of an image or a subset of the image for better contrast or visualization of image features and to facilitate more accurate subsequent image analysis.

The spatial domain methods accomplish image enhancement using either global operations on the whole image or local operations on a neighborhood region of each pixel. The operations used to increase contrast in the image include contrast stretching, clipping and thresholding, image subtraction and averaging, and histogram equalization and specification. The operations used to sharpen image features and reduce noise include spatial band pass filtering, directional and steerable filtering, and median filtering. If image noise is a random stationary process, variants of the Wiener filter can be used to reduce the noise effectively. Nonlinear filters, such as the median filter, can reduce noise without blurring edges.

Transform domain methods accomplish image enhancement based on computations performed in a transform domain, such as the Fourier or wavelet transform. Often, salient image features can be more easily isolated and extracted in the transform domain than in the spatial domain. Widely used Fourier domain image enhancement methods consist of Wiener filtering, least-squares deconvolution, and band pass filtering. The Wiener filter is most helpful for noise removal in the sense of lowest mean square error. Wavelet domain image enrichment methods influence the advantages of multiscale image representation and nonlinear filtering. Since image edges tend to correlate spatially across multiple scales whereas noise does not, one can exploit this property and use nonlinear filtering to accentuate edge structures effectively while suppressing noise in the image. Basically all of the techniques developed for the enhancement of monochrome images can be applied to enhance color images by performing the operations 
on their intensity or luminance component, where image contrast and edge information is encoded. Figure 4 shows different group of techniques, image enhancement algorithms can be developed to accomplish the image quality for further analysis. The mathematics behind these techniques is same as it is in the conventional image processing [3].

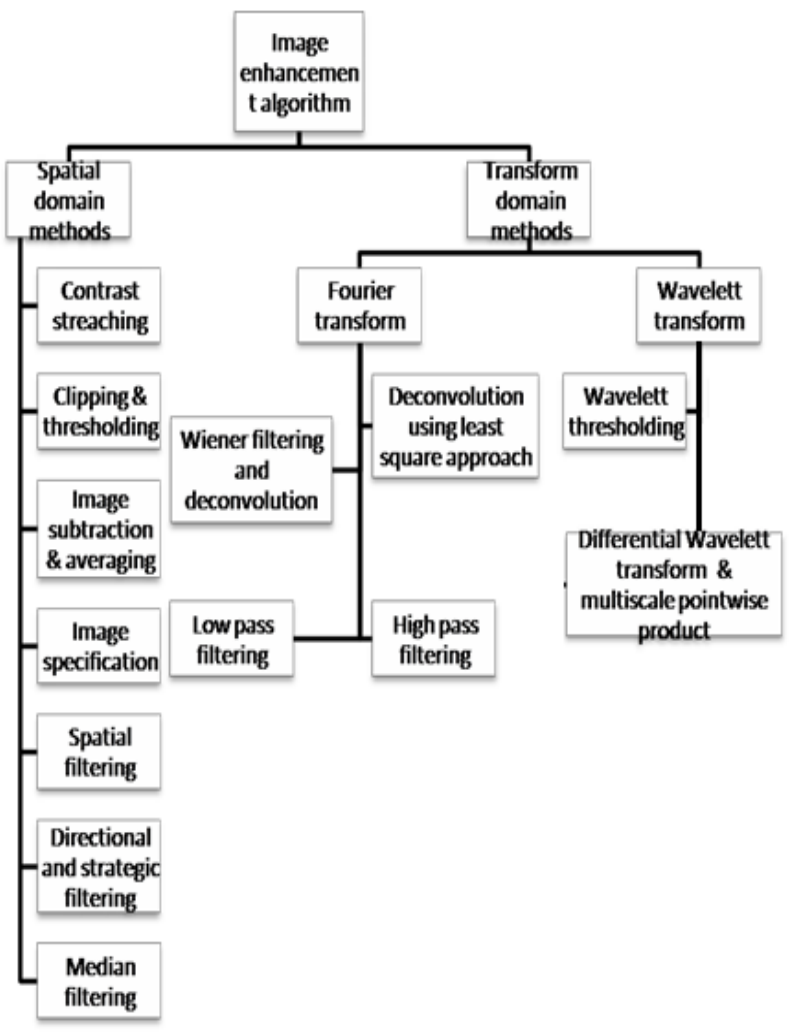

Figure 4: Category of Image Enhancement Techniques

\subsection{Wavelet Image Processing}

Multi resolution image representations using wavelet transforms have become quite accepted in recent years, owing to their effectiveness in a very broad array of applications. In essence, wavelets made it possible to formalize the general concept of multi resolution processing that was being used, for example, in the computer vision field to enable to detection, analysis, and recognition of image features and objects over varying ranges of scales. Such important image processing tasks as segmentation require that the image be analyzed over neighborhoods of varying sizes in order to capture salient image features and properties that occur at different scales.

Nowadays, in a remarkable variety of image processing applications, the discrete wavelet transform has become the indispensable formal mathematical tool for creating and manipulating multi resolution representations [3]

\subsection{Morphological Image Processing}

Morphological processing has applications in such different areas of image processing as filtering, segmentation, and pattern recognition, to both binary and grayscale images. One of the advantages of morphological processing is its being well suited for discrete image processing, because its operators can be implemented in digital computers with complete fidelity to their mathematical definitions. Another benefit of morphological processing is its inherent building block structure, where complex operators can be created by the composition of a few primitive operators.
Morphological image processing is based on probing an image with a structuring element and either filtering or quantifying the image according to the manner in which the structuring element fits (or does not fit) within the image. Erosion and dilation are primitive operators of morphology processing, and they are duals. The erosion of an image consists of all locations where the structuring element fits inside an object. Opening and closing are created from the composition of erosion and dilation. They are dual operations. An image contains its opening, whereas it is contained by its closing. The opening of an image by a structuring element is the union of all the structuring elements that fit inside the objects. It is a way of marking objects that have certain specified morphological properties.

In grayscale operations the image is depicted in four different graphical representations: (a) the pixel values mapped in gray levels (low values are dark and high values are bright gray tones); (b) the pixel values also mapped in grayscale but in a reverse order; (c) the same image but as a top view of a shaded surface; and (d) a mesh plot of the same surface.

Grayscale erosion (or dilation) by a flat structuring element is equivalent to a moving-minimum (moving-maximum) filter over the window defined by the structuring element. Grayscale erosion, dilation, opening, closing, ASF, morphological reconstruction, alternating sequential component filters, area opening, and closing are stack filters. They can be implemented by operating on the threshold sets with their binary-equivalent operator, followed by stack reconstruction [3].

\subsection{Image segmentation}

Image segmentation is a task of fundamental importance in digital image analysis. It is the process that partitions a digital image into disjoint (non-overlapping) regions, each of which typically corresponds to one object. Once isolated, these objects can be measured and classified Image segmentation is usually approached from one of two different but complementary perspectives, by seeking to identify either the regions or the boundaries of objects in the image.

A region is a connected set of (adjacent) pixels. In the regionbased approach, we consider each pixel in the image and assign it to a particular region or object. In the boundary-based approach, either we attempt to locate directly the boundaries that exist between the regions or we seek to identify edge pixels and then link them together to establish the required boundaries. Segmentations resulting from the two approaches may not be exactly the same, but both approaches are useful to understanding and solving image segmentation problems and their combined use can lead to improved performance. Regionbased techniques partition the image into sets of interior and exterior pixels according to similarity of image properties. Boundary-based techniques establish object boundaries by detecting edge pixels that are associated with differences in image properties. Variations and combinations of the basic techniques presented here often must be tailored to the specific application to produce acceptable results.

Thresholding is an essential region-based image segmentation technique that is mainly useful for scenes containing solid objects resting on a contrasting background. It is computationally simple and never fails to define disjoint regions with closed, connected boundaries. The operation is used to differentiate between the objects of interest (also known as the foreground) and the background on which they lay. The output is either the label "object" or "background," which can be represented as a Boolean variable. 
Gray-level thresholding is a simple region-based segmentation technique. If not the background gray level and object contrast are relatively constant, it is usually necessary to vary the threshold within the image. This is adaptive thresholding. The selection of the threshold value is crucial and can significantly affect the boundaries and areas of segmented objects. For images of simple objects on a contrasting background, placing the threshold at the dip of the bimodal histogram minimizes the sensitivity of object area measurement to threshold variation. Both the profile function of a concentric circular spot and the average gradient around a contour line can be derived from the histogram or from the perimeter function of its image.

Morphological processing can improve the initial segmentation results from thresholding by using procedures such as separation of touching objects and filling of internal holes. Unlike thresholding, region growing and splitting techniques exploit the spatial context in complex scenes. Region growing combines adjacent regions into larger regions, within which the pixels have similar properties. Region splitting partitions larger regions into smaller adjacent regions, within which the pixels have different properties.

Edges correspond to the image points where gray level changes abruptly, and they usually occur on object boundaries. Edge points can be detected and used to establish the boundaries of objects. Gradient-based methods detect edges by looking for the pixels with large gradient magnitude. Laplacian-based methods search for zero-crossings in the second derivative of the image to find edges.

Object boundaries can be established by thresholding either the gradient image or the Laplacian image if edges are strong and the noise level is low. The detected edge points seldom form closed connected boundaries that are required for image segmentation. Therefore edge linking and boundary refinement are usually performed to complete the object boundary delineation process. The Hough transform can fit a parameterized boundary function to a scattered set of edge points. Active contours can be used to filter boundaries that have been found by other methods. The outcome of image segmentation can be encoded and stored conveniently either as an object label map or as a boundary chain code.

Real-world applications in digital microscopy frequently pose very challenging segmentation problems. Variations and combinations of the basic techniques presented here often must be tailored to the specific application to produce acceptable results.

There is no common approach to obtain accurate image segmentations; more or less all techniques come together the two main schemes such as region based and edge based. The majority used methods and how they are usually combined to achieve good segmentation results. Which is helpful for better use of existing method and for improving the performance as well as for designing new ones [3] [4].

\subsection{Object measurement}

The basic objectives of object measurement are application dependent. It can be used simply to provide a measure of the object morphology or structure by defining its properties in terms of area, perimeter, intensity, color, shape, etc. It can also be used to discriminate between objects by measuring and comparing their properties. Object measurements can be broadly classified as (1) geometric measures, (2) ones based on the histogram of the object image, and (3) those based on the intensity of the object. Geometric measures, including those that quantify object structure, can be computed for both binary and grayscale objects. In contrast, histogram- and intensitybased measures are applicable to grayscale objects. Another category of measures, which are distance based, can be used for computing the distance between objects or between two or more components of objects. Common measurements of both gray scale and binary objects are describe in terms of

\section{Size measure}

The size of an object can be defined in terms of its area and its perimeter. Area is a convenient measure of overall size. Perimeter is particularly useful for discriminating between objects with simple shapes and those with complex shapes.

\section{Pose measure}

The pose of an object is typically defined by its location and orientation. Measuring its centroid can indicate the location of an object. Object orientation is normally measured by computing the angle subtended by its major axis.

\section{Shape measure}

Shape measures are increasingly used as features in objectrecognition and objects -classification applications to distinguish objects of one class from other objects. Shape features are generally invariant to translation, rotation, and scaling. These features can be used independent of, or in conjunction with, area and perimeter measurements. Commonly shape parameters are thinness ratio, rectangularity, circularity, Euler numbers, moments, elongation.

A shape descriptor is another way of describing an object's shape. It provides a more detailed description of shape than that offered by the single parameter shape measures. The most common shape descriptors include the boundary chain code (BCC) and its derivative known as the differential chain code (DCC). Fourier descriptors exploit the periodicity in the BCC representation of the boundary. Medial axis transformation (MAT) is another data reduction technique that is used as a shape descriptor. Objects can be described using compact descriptors such as a chain codes, the medial axis transform, and graphs. Graphs have been used as a tool for translationand rotation-invariant representation of object shapes.

\section{Distance measure}

Measures of distance provide a way to compute the separation between two points in an image. These can be two points within the same object (such as points on the major and minor axes) or points on two different objects. The three most common ways of measuring distance are Euclidean distance, City-block distance It is also called Manhattan distance or the absolute value metric, chessboard distance.

Object measurements derived as a function of the intensity distribution of the object are called gray-level object measures. There are three foremost categories of gray-level object measurements. Intensity and histogram measures are normally defined as first-order measures of the gray-level distribution, whereas texture measures quantify second- or higher-order relationships among gray-level values. Histogram measures capture the statistics of an object's gray levels. Texture measures capture the statistics of an object's gray-level structure [3].

\subsection{Object classification}

Classification is the step that tells us what is in the image. Assuming the objects in the image have been segmented and measured, classification identifies them by assigning each of them to one of several previously established categories or classes. Here we illustrate the process of classification with the very useful maximum-likelihood method. This technique is 
widely used because it minimizes the probability of making an incorrect assignment. More specifically, we present the minimum Bayes risk classifier, assuming Gaussian statistics.

\subsubsection{The classification process}

When we encounter an object in a microscopic image, we know three things about it. First, the a priori probability that it belongs to each of the classes. Second, the objects measured feature values. This is the quantitative data that is unique to that particular object. Third, probability density function (pdf) of those features for each of the classes. This specifies what is known about each class. Given these three pieces of knowledge, to make an optimal assignment of that object to a class. Combining these three things one can be acquainted with about an object to find its most likely class. After an object has been measured, one can able to use the measurement data and the class-conditional pdfs to improve our knowledge of the object's most likely class membership. The posteriori probability that the object belongs to class - I is given by Bayes' theorem; it then, allows combining a priori probabilities of class membership with the measurement data and the classspecific pdf to compute the probability that the measured object belongs to each class. Given this information, we can assign each object to its most likely class.

The objects can be classified as per the following cases

- $\quad$ The Single features Two-Class case

- $\quad$ The Three-Feature ,Three-Class Case

Once a classifier has been designed and trained, it is necessary to test it to establish its accuracy. This is usually done by classifying a test set of known objects and tabulating the number of errors. If the test set is the same as the training set, the performance estimates will be optimistically biased. If it includes none of the training data, they will be pessimistically biased. If the test set is large, the effects of this bias will be slight. The confusion matrix is the tools that are used to measure the performance of classifier accuracy. A well-trained Bayes classifier can be quite effective at multiclass, multifeature classification, even in the presence of considerable noise. One should pay particular attention to numerical precision issues since some of the parameters in the probability calculations can become quite large or quite small. A nonlinear transformation can make a feature's pdf symmetrical. A multimodal pdf suggests the presence of subclasses. Judicious use of sub classing and feature transformations can often make the Gaussian assumption work. When a particular functional form for the pdf (the Gaussian, for example) is known, less training data is required since it is used only to estimate the parameters. This gives rise to a parametric classifier. If the functional form of the pdf is not given or it is known to be nonGaussian, one must estimate the pdfs directly from the training data. Such classifiers are nonparametric, and they usually require considerably more training data.

When available training samples are inadequate to estimate the pdfs accurately, one can choose independent features by mapping the original features using a proper transformation such as independent component analysis. With this method the problem of estimating a multivariate pdf is simplified to that of estimating multiple univariate pdfs, thereby considerably reducing the size requirements of training sets.

As the number of features increases, the dimensionality has been increased and hence the volume of the feature space. This, in turn, increases the requirements for training set and test set size. Adding features that have poor discrimination or are highly correlated with the other features can actually degrade classifier performance. To reduce the features, there are welldeveloped mathematical procedures for reducing a large number of features down to a smaller number without severely limiting the discriminating power of the set. For reducing a large number of features down to a smaller number without losing their discriminating power, Principal component analysis (PCA) and linear discriminant analysis (LDA), also known as Fisher discriminant analysis (FDA) are two wellknown techniques. PCA uncorrelated the new features and maximizes their variance, whereas LDA maximizes the ability of the features to discriminate among the classes.

The use of artificial neural networks (ANNs) is a different approach for classification. An ANN classifier has the advantages that it is not necessary to know the statistics of the features in order to function, and the decision surfaces it can implement in feature space are more complex than the secondorder surfaces that the parametric Bayes classifier generates. However, its disadvantages are that it is a "black box" and it is difficult to prove optimality or to predict error rates. It also lacks the rich analytical underpinning that supports the design of statistical classifiers [3] [4].

\section{APPLICATIONS OF MIP}

Image processing techniques have been widely used in the last decade in medical imaging and the microscopic field received a consistent effort from researchers. The microscope image processing techniques are of special interest because they allow large scale statistical evaluation in addition to classical eye screening evaluation and are used in various fields from medical to physical structures of the things.

\subsection{Medical sciences}

In the medical field the microscopic examinations have sections of the pathology: cytology (the study of cells) and histology (anatomical study of the microscopic structure of tissues). Considering the importance of the pathological results for human health and the applications difficulties, many computer aided image analysis systems have been proposed. The complex diagnostic process (time consuming and tedious process) can be enhanced by providing the pathologists or the biologists with quantitative data extracted from the images like blood cell, semen sample, embryo , DNA with staining, tumor tissue as shown in figure 5. Most of the pathological laboratories are doing cell analysis, semen analysis by setting up the video setup with microscope or semen analyzers. [5]

By using some microscope image processing techniques result of finding the individual spermatozoa and so concentration of semen sample can be improved [6]. The lamina A/C fluorescent staining is used to visualize the DNA. After detecting the clusters of cells nuclei, the significant concavity points are detected. A set of geometrical templates use these points to detect the aggregated and the overlapped nuclei configurations. Another area is leukocytes segmentation in images of bone marrow sample. Morphologic examination of bone marrow is the cornerstone in diagnostic hematology.

\subsection{Agriculture and botany}

In agriculture to find the diseases on plants and in the field of botany as shown in figure 6 , to study the biologists to examined living plant cells . 


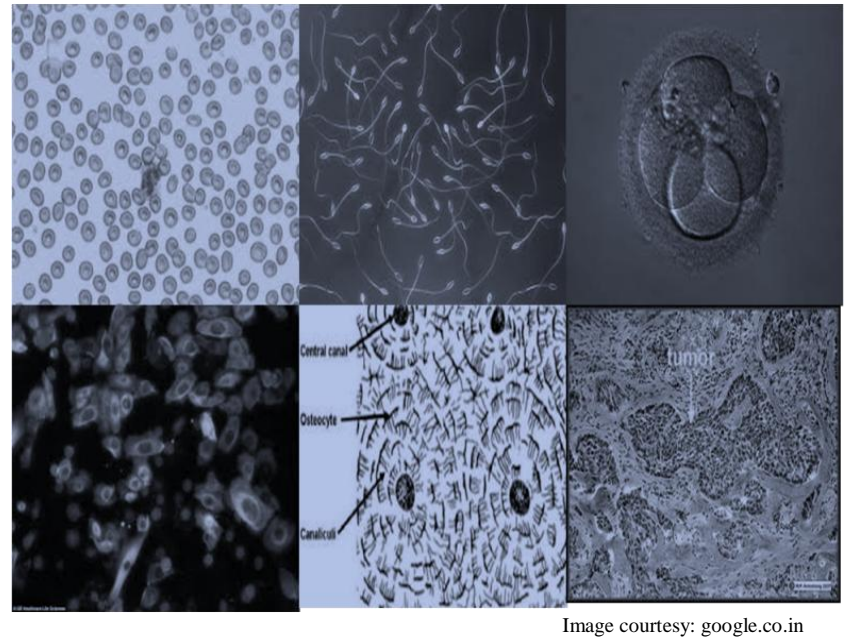

Figure 5: Microscopic view of blood cell, semen, embryo, DNA with staining, tumor tissue

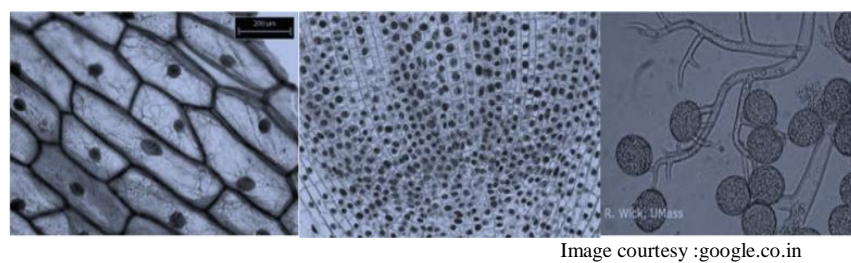

Figure 6: Microscopic view of living plants cell, diseases

\subsection{Microstructure of materials in metallography}

The application of microscopy for assessing the properties of materials has long history. Microstructure refers to the microscopic description of the individual phase constituents of a material. The microstructure images are the images of materials observed at a micro-scale level. These images determine the properties of material formed as a result of solidification processes during the manufacturing stage. It is difficult to put sensors at all positions within a big casting. Furthermore, a considerable amount of time needs to be spent on quantitative metallography to study the material which involves cutting samples, thorough polishing, etching and finally image analysis.

Some of microstructure images of cast iron are shown in figure 7 [7][8].

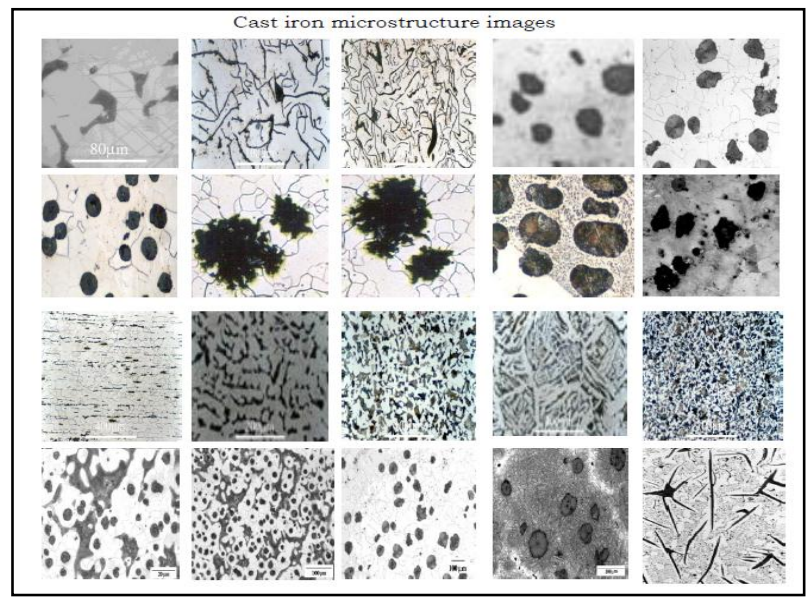

Figure 7: Microstructure images of cast iron

\section{CONCLUSION}

The increasing use of Microscope image processing (MIP) has seen increasingly used in the field of biomedical research and clinical medicine as well as in many other fields. This helps the stakeholders to take decisions in better fashion. Resulting as Microscope Image Processing emerged as a prominent area of research in the last decade. One can easily apply the basic and advance concepts of Digital Image Processing (DIP) in this field. It can be applicable in diverse field like medical imaging; bioinformatics etc. Microscope image processing allow large scale statistical evaluation in addition to classical eye screening evaluation and are used in various fields from medical to physical structures of the things. Also the use of this research may lead in the economical solutions to the society and can be widely accepted by anyone without having any expertise in the field of image processing. This opens a vast area for the researcher in the field of image processing.

\section{REFERENCES}

[1] "Microscope_image_processing" Available www.en.wikipedia.org Accessed 17 October 2010.

[2] "Measuring with microscope" Available at www.ruf.rice.edu Accessed 10 December 2014.

[3] Qiang Wu, Fatima A. Merchant, Kenneth R. Castleman, "Microscope Image Processing", Elsevier Publication, ISBN: 978-0-12-372578-3.

[4] Cristian Smochina, Paul Herghelegiu ,Vasile Manta, "Image Processing Techniques Used In Microscopic Image Segmentation”, June 2011.

[5] Chaudhari, N.M., Pawar, B.V., "Light scattering study on semen analysis methods/techniques," Engineering (NUiCONE), 2013 Nirma University International Conference on Engineering, vol., no., pp.1,4, 28-30 Nov. 2013.

[6] Chaudhari, N.M., Pawar, B.V, "New hybrid approach for identification of spermatozoa in human semen sample using microscope image processing techniques", Advances in Image and Video Processing, volume 2, Issue 6 , ISSN:2054 with DOI: 10.14738,15-24, December 2014 .

[7] P S Hiremath, Anita Sadashivappa and Prakash Pattan. ,"Article: Microstructure Image Analysis for Estimating Mechanical Properties of Ductile Cast Iron", International Journal of Computer Applications 107(17):32-35, December 2014 .

[8] Prakash Pattan , "Analysis and characterization of microstructure images of materials using digital image processing techniques", Ph.D. dissertation, Department of computer Science, JNTU, Hydrabad, 2011 